\title{
Réseaux et marchés en Chine : la réorganisation de l'industrie sucrière face à la mondialisation ${ }^{1}$
}

\author{
Networks and Markets in China: \\ the Reorganization of the Sugar Industry \\ in the Event of Globalization
}

\author{
Louis Augustin-Jean
}

\author{
Graduate School of Public Policies and Modern Culture \\ Department of Public Policies, University of Tsukuba \\ 1-1-1 Tennodai, Tsukuba-Shi, Ibaraki-ken 305-0069, Japan
}

\section{Résumé}

L'entrée de la Chine dans l'OMC a souligné l'importance de ce pays dans les échanges économiques internationaux; les réformes de libéralisation qu'il a introduites sont potentiellement lourdes de conséquences sur l'évolution des marchés mondiaux, en particulier pour les produits agricoles. L'article s'attache donc à présenter la logique des réformes chinoises pour un produit agricole majeur, le sucre, dont le commerce est marqué par la prégnance d'accords internationaux. Il est montré que la mise en place des réformes a donné lieu à des distorsions importantes au niveau local, en fonction des intérêts divergents des acteurs. Il est alors clair que les mesures libérales ont été détournées en fonction des jeux de pouvoirs existants dans l'industrie, et d'une opposition entre une logique bureaucratique et une logique de marché qui peine à s'imposer. Bien que la Chine soit le $3^{\mathrm{e}}$ producteur mondial de sucre et que sa consommation soit en progression constante, la survie de cette industrie passe par la résolution de cette opposition.

(c) 2007 Lavoisier, Paris. Tous droits réservés.

\footnotetext{
${ }^{1}$ Certains éléments de ce texte ont été publiés en «note de recherches » dans Économies et Sociétés, série SAA, $\mathrm{n}^{\circ} 28$, sous le titre, «Les stratégies des agents et l'organisation du marché du sucre en Chine dans un contexte de mondialisation ».

*Adresse email : auyeung_ly@yahoo.fr

doi:10.3166/ges.9.439-461 (C) 2007 Lavoisier, Paris. Tous droits réservés.
} 


\begin{abstract}
Summary
The entry of China into the WTO underlined the importance of this country in the international trade. The liberalization reforms that she introduced can lead to major consequences in the evolution of the world markets, especially in relation to food products. Therefore, this article is devoted to present the logic of Chinese reforms for an important commodity, sugar, which trade is characterized by major international agreements. It is shown that the introduction of reforms lead to important distortions at the local level, in relation to the divergent interests of the actors within the industry. It is then clear that some measures of liberalization have been distorted, due to the power relationships that exist within the industry and to on an opposition between a bureaucratic and a market logic. Even though China is the $3^{\text {rd }}$ largest world producer of sugar and her consumption is constantly increasing, the survival of this industry implies a resolution to this opposition.

(C) 2007 Lavoisier, Paris. Tous droits réservés.
\end{abstract}

Mots clés : sucre, développement local, économie de la Chine, mondialisation, organisation du marché

Keywords: sugar, local development, China economy, globalization, market organization.

En 2001, la République populaire de Chine (RPC, ou Chine), entrait dans l'OMC, après des années d'intenses négociations. Le mythe du milliard de consommateurs, le poids de ce pays dans les échanges mondiaux ou comme récipiendaire d'investissements directs étrangers rendaient son adhésion à cette organisation obligatoire, pour elle comme pour ses partenaires commerciaux.

Pourtant, cette position nouvelle au sein d'un ordre économique mondial à dominante libérale n'est pas sans poser un certain nombre de questions, qui expliquent d'ailleurs la longueur des négociations qui ont préludé à son entrée dans l'organisation. Malgré des années de changements, l'économie chinoise reste à tendance socialiste, et l'adéquation entre son mode de fonctionnement et celui de l'OMC n'est pas un débat uniquement théorique. Les accords signés par la Chine avec ses partenaires commerciaux nécessitent ainsi un nouveau cycle de réformes, que le gouvernement central est tout disposé à mettre en place dans le respect des règles établies par l'OMC. Cependant, l'application de ces réformes ne dépend pas uniquement du gouvernement, mais surtout du bon vouloir des autorités locales, dans un contexte où le marché chinois demeure largement fragmenté. Comme dans d'autres pays, les bases de la redistribution des pouvoirs entre les niveaux supranational, national et local sont donc en cours de redéfinition. L'action du gouvernement central semble affaiblie par les règles de plus en plus contraignantes de la globalisation de même que par les pratiques locales, la décentralisation des années 1980 ayant entraîné un contrôle grandissant des autorités locales sur leur politique économique.

Ces questions sont parfaitement illustrées par l'industrie sucrière. Ainsi, alors que la réunion interministérielle de l'OMC, à Cancun, a mis en exergue les positions a priori inconciliables entre les tenants de la libéralisation agricole et ceux qui y sont opposés - ce qu'a confirmé la réunion de Hong Kong -, la Chine a entamé, dès le milieu des années 1990, des réformes majeures de ses IAA, visant à introduire une certaine dose d'économie de marché - afin, justement, de préparer son adhésion à l'Organisation. 
C'est dans cette atmosphère qu'à partir de 1997 s'est engagée la transformation de l'industrie sucrière. La Chine socialiste est donc devenue l'un des premiers pays à déréguler cette industrie, dans un marché mondial largement régulé par des accords internationaux - ce qui fait dire à de nombreux responsables chinois qu'elle a été sacrifiée sur l'autel de la mondialisation. Pourtant, la production a connu une progression presque linéaire ces dernières années, et se situe aujourd'hui autour de 10 millions de tonnes. La Chine est le troisième producteur mondial, derrière le Brésil et l'Inde. Sa production est concentrée dans la province du Guangxi ${ }^{2}$, située dans le sud-ouest tropical du pays : plus de la moitié du sucre chinois y est fabriqué, à partir de la canne cultivée localement ${ }^{3}$.

Cet article cherche donc à analyser les changements récents survenus dans la filière sucre dans le Guangxi, dans le contexte de dérégulation qui vient d'être rappelé. Ces changements se sont produits sous la pression de forces externes (mondialisation, «consensus de Washington ») et de forces internes (crise du modèle d'accumulation conduisant à la nécessité de réorganiser la production au niveau local). Cela a abouti à la recomposition des réseaux locaux et, en conséquence, à la réorganisation spatiale de la province. De façon classique, les changements sont donc provoqués par la crise du régime d'accumulation fordiste (Benko, 1990, p. 17). Au niveau international, le sucre, comme l'ensemble des matières premières, se caractérise par une forte volatilité des prix, due à des périodes de surproduction faisant suite à des périodes de forte demande (Saadi, 2005), en relation avec la situation de l'économie mondiale. Depuis les années 1970, on constate également une corrélation inverse forte entre l'offre de sucre et le prix du pétrole, en partie du fait de la mise en place par le Brésil du «plan éthanol » (Audinet, 1998) ${ }^{4}$.

Pourtant, le sucre étant un produit de production (et de consommation) de masse par excellence - ce que reflète parfaitement le terme anglais de «commodity »-, la réorganisation spatiale ne peut s'effectuer suivant le modèle devenu classique (même s'il est toujours débattu) de la production flexible post-fordiste. Ainsi, alors que le sucre constitue une composante majeure de l'économie du Guangxi, la question de la réorganisation d'une industrie de production de masse dans un cadre soumis à la poussée de forces postfordistes doit être posée, et les contradictions qui peuvent surgir dans ce schéma (voir page suivante) particulier doivent être mises en avant.

L'analyse doit alors porter sur la recomposition territoriale, comprise de façon abstraite par la redéfinition des réseaux. C'est ainsi qu'il est montré, dans cet article, comment le sens originel des réformes a été perverti par l'action de (et l'interaction entre) différentes catégories d'agents, dépendantes de leur position au sein du système et des intérêts divergents qu'ils défendent. Cet article ne constitue donc pas une analyse filière classique ${ }^{5}$, mais

\footnotetext{
${ }^{2}$ Cf. carte 1 en annexe.

${ }^{3}$ La province est appelée officiellement « Région autonome Zhuang du Guangxi ». Elle constitue l'une des 31 unités administratives chinoises ayant le rang de province.

${ }^{4}$ Par exemple, le prix du sucre dans les années 1973-1974 sur les marchés internationaux a atteint 60 US cents par livre. Au début des années 2000, il était tombé à 9 cents, pour dépasser 21 cents en 2005.

${ }^{5}$ Des passerelles seraient cependant intéressantes avec l'approche filière française ou la commodity value chain anglo-saxonne. Sur ce point, voir Gereffi (1999) et Kaplinski (2000), ainsi que l'ouvrage récent de Daviron et Ponte (2006).
} 
la perspective systémique qui est proposée s'inscrit dans la continuité des dynamiques territoriales (Pecqueur, 1996; Benko, 1990) et de l'économie de la proximité (Rallet et Torre, 2004). Cette analyse procure en outre une description du mode d'organisation économique chinois, tel qu'il émerge après plus de 25 années de réformes. L'article est divisé en deux parties. S'inscrivant dans une problématique territoriale, la première présente un état des lieux de la production sucrière du Guangxi et montre pourquoi les réformes étaient devenues indispensables à partir du début des années 1990. La seconde partie se place dans une perspective monographique et empirique, et présente les réformes engagées et leurs effets. Cette seconde partie est basée plus particulièrement sur deux études de cas; la première porte sur les réformes entreprises sur un groupe étatique et la seconde sur le mode de privatisation engagée dans l'industrie. Ces deux études de cas ont été réalisées dans le cadre d'un travail de terrain et d'une collecte documentaire effectués lors de six séjours d'une période de deux semaines chacun, situés entre avril 2004 et mars 2006. Ils apparaissent significatifs des réformes engagées par la Chine - en particulier sur son industrie sucrière mais pas uniquement - et seront appuyés ponctuellement par d'autres données.

\section{La difficile maturation d'une industrie en croissance}

\subsection{Prologue : marché et problématique territoriale}

En 1978, au moment où la Chine, émergeant de 30 années de maoïsme, lance son programme de réformes économiques, la production de sucre n'était que de 2,27 millions de tonnes, dont seulement 251000 tonnes pour la province du Guangxi (soit environ $11 \%$ ). En 2005, elle s'élevait à 9,12 millions de tonnes, dont 5,04 millions pour le seul Guangxi (55,3\%) (cf. Figure 1).

Fig. 1 : production de sucre en Chineau Guangxi, 1978-2005

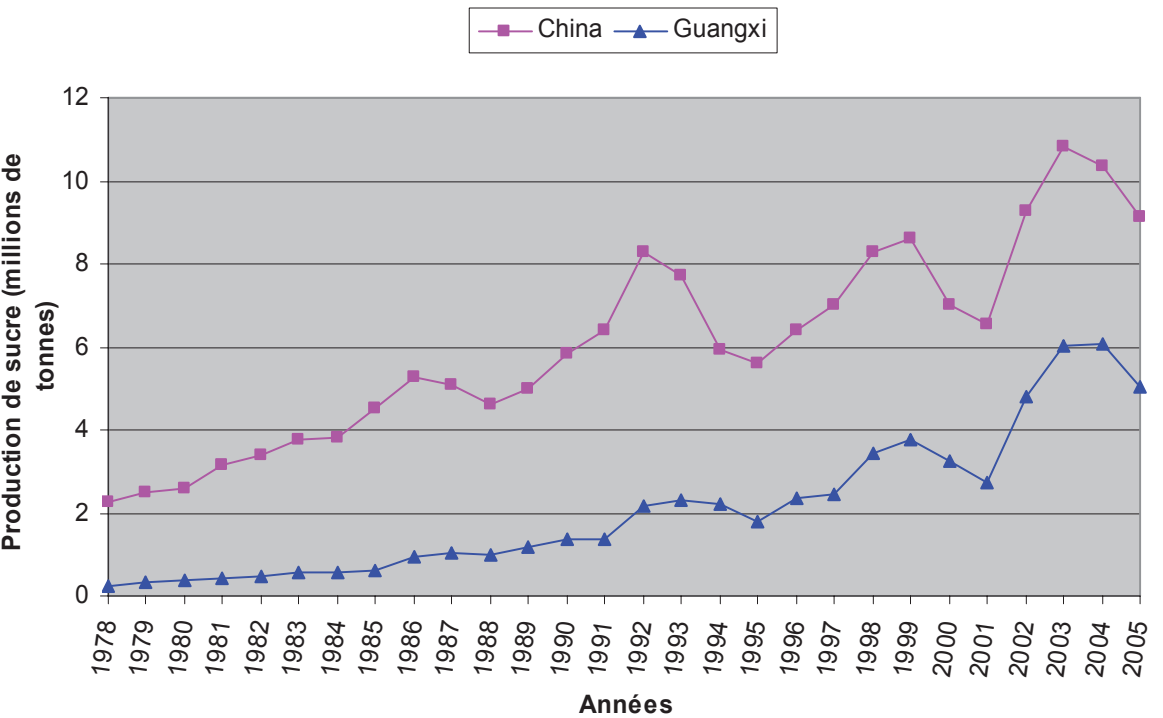

Sources : Guangxi Tongji Nianjian (Annuaire Statistique du Guangxi), diverses années ; China Statistical Yearbook, diverses années. 
Dans l'intervalle, la consommation a augmenté significativement, pour se situer aujourd'hui entre $7 \mathrm{~kg}$ et $10 \mathrm{~kg}$ par habitant. Cette augmentation devrait se poursuivre, au moins sur le moyen terme ${ }^{6}$.

Durant les 25 dernières années, s'est donc constitué un véritable marché du sucre, alimenté par une augmentation parallèle (mais non simultanée) de la production (de plus en plus concentrée dans le Guangxi) et de la consommation (surtout dans les grandes villes et les régions côtières). Il y a ainsi une opportunité, presque unique, d'étudier le mode de fonctionnement d'un marché en construction, à partir de la mise en place des réformes engagées à la fin des années 1990 et de ses effets. La démarche est donc empirique : il s'agit d'ouvrir les «boîtes noires » des entreprises et du marché, afin d'étudier leurs formes de coordination au sein d'un cadre spatial. Dans ce sens, le cadre d'une province comme le Guangxi est particulièrement pertinent, puisque que cette dernière forme un centre administratif et une unité de décision bien délimités : la province constitue depuis des siècles le rouage administratif du gouvernement central, en charge de l'implantation des politiques économiques; elle doit aussi rapporter les problèmes et les plaintes des districts et des populations locales au gouvernement central. Il s'agit donc d'un espace bien articulé, ayant une épaisseur historique et culturelle, et dans lequel s'articulent des réseaux plus ou moins denses.

La réflexion s'élabore alors autour de la conjonction entre un paradigme technologique (issu de la contrainte de croissance de la production et d'économies d'échelle propres à un produit standard) et un paradigme organisationnel qui, de façon formelle ou informelle, définit le mode de coordination des acteurs (Crévoisier, 2004). Le paradigme territorial se déduit alors de trois dimensions distinctes. Premièrement, de la conjonction entre les deux premiers paradigmes; deuxièmement, du niveau de concurrence et de collaboration qui existe non seulement entre les firmes, mais aussi entre des sous unités territoriales bien définies; troisièmement, de la concurrence entre l'espace territorial structuré et d'autres espaces territoriaux. Le cadre territorial est ainsi porteur de dynamiques pouvant générer des ressources et des savoir-faire, mais aussi des phénomènes de « concurrence destructive » et des blocages?

Le cadre territorial n'est donc pas seulement défini par la simple délimitation administrative et organisationnelle et par son épaisseur historique. Il est également

\footnotetext{
${ }^{6}$ Avec une population de 1.3 milliards de personnes, une consommation par habitant de $7 \mathrm{~kg}$ (CNUCED, site internet) ou de $9 \mathrm{~kg}$ (USDA, 2005) a une conséquence importante sur la consommation totale. Selon l'estimation la plus basse, la consommation totale est de 9,1 millions de tonnes, et la Chine est alors surproductrice. Dans le second cas, elle doit recourir aux importations. Par ailleurs, la consommation par tête, qui était de $3,9 \mathrm{~kg}$ en 1978, est en progression constante, mais reste inférieure à celle de nombreux pays, y compris des PED : Vietnam, 11,1 kg; France, 34,3 - ces deux chiffres étant pour l'année 1998 (CNUCED, site internet).

${ }^{7}$ La définition est ainsi sensiblement plus large que celle donnée par Crévoisier (2004). Faute de place, la concurrence entre espaces territoriaux ne sera pas analysée en détail dans cet article. En bref, cette dernière s'exprime à travers deux dimensions majeures : premièrement, à travers les paramètres expliquant comment le Guangxi est devenu le plus grand producteur de sucre du pays - et le plus compétitif -, au détriment de producteurs traditionnels tels que le Guangdong et le Fujian; deuxièmement, à travers les facteurs internes et externes qui montrent comment la province réussit à maintenir ses nouveaux compétiteurs (le Yunnan par exemple) à distance. À côté des dimensions historiques, culturelle et administrative, la concurrence entre territoires joue donc un rôle de premier plan dans la construction du paradigme territorial - rôle qu'il est important de garder à l'esprit.
} 
constitué de réseaux et de relations de collaboration et de concurrence entre les firmes et entre des sous-ensembles territoriaux (par exemple, dans le cas chinois, entre les districts, qui ont également une épaisseur historique importante $)^{8}$. Il est renforcé par les contraintes technologiques qui découlent des capacités de production internes, des politiques publiques (internes et externes), de la demande et de la concurrence territoriale externes. Lorsque le marché est en constitution, comme dans la province du Guangxi, ces différentes forces agissent parfois en sens contraire, et leurs effets deviennent particulièrement visibles de par l'action des acteurs. Une démarche empirique visant à comprendre ces actions devient alors justifiée (Latour, 2005) - l'enjeu étant de savoir si les changements en cours peuvent aboutir à l'établissement de paradigmes viables et à la mise en place d'un milieu, sinon innovateur, du moins promouvant le développement. Comme nous allons le voir, cela est largement dépendant de la forme prise par le marché et de sa viabilité, ainsi que de l'action des acteurs au sein d'un territoire en évolution.

La perspective qui consiste à examiner l'organisation économique d'une industrie ou d'une filière dans un cadre géographique précis se démarque donc nettement des analyses habituelles sur l'économie chinoise, qui supposent plus ou moins explicitement que ce pays se dirige vers l'économie de marché. De façon plus positive, elle dépasse aussi l'alternative organisation/marché (Williamson, 1985), et considère ce dernier comme un type spécifique d'organisation. Le marché ne se définit plus comme un point (spot) de rencontre entre l'offre et la demande, ni même comme une construction sociale dépendante du milieu dans lequel elle se situe : s'il y a bien un « encastrement» (Granovetter, 1985) du marché dans l'organisation sociale, ce dernier forme aussi une organisation (sociale) à part entière dont le fonctionnement influe sur le mécanisme de fixation des prix (autrement dit, sur la performance du marché). Dans un environnement caractérisé par de nombreux risques et incertitudes et des coûts de transaction importants (comme c'est le cas en Chine), la structure du marché s'organise autour de réseaux qui permettent aux échanges de s'effectuer : elle offre une alternative à l'impersonnalité du marché comme au renforcement de la hiérarchie. Ces réseaux, formels ou informels, relient non seulement les entreprises entre elles, mais aussi entreprises et administrations et, au-delà, les individus et les objets (Callon, 1998). Ils sont donc au cœur du paradigme organisationnel, tel qu'il a été défini précédemment.

Les réseaux sont donc multiformes et offrent la possibilité aux agents de prendre des décisions en situation d'incertitude. Ce qui importe alors n'est plus de savoir si les entrepreneurs doivent arbitrer entre le marché ou la hiérarchie, mais quel genre de réseaux ils sont capables de développer ou de mobiliser. Selon les mots de Philippe Hugon, dans un environnement risqué et incertain, "la prise de décision constitue un premier niveau de coordination » (Hugon, 1995). Il faut donc penser un marché non plus impersonnel mais défini autour de ces réseaux (qui structurent par ailleurs le territoire), et comprendre ses éventuels blocages en relation avec les stratégies des agents, en relation avec leur position au sein du système.

\footnotetext{
${ }^{8}$ Les divisions administratives chinoises suivent ce modèle général, avec cependant des variations innombrables dans le détail : État $\rightarrow$ Provinces $\rightarrow$ Municipalités $\rightarrow$ Districts $\rightarrow$ Bourgs et Cantons. Dans cet article, le terme « autorités locales » s'applique aux districts et aux divisions administratives inférieures.
} 
Concrètement, il s'agit d'examiner, au sein de la filière sucre, comment les principaux agents - paysans, autorités locales et entreprises -, mus par des intérêts et des positions systémiques divergents, s'organisent dans l'espace géographique de la province, en relation avec les changements institutionnels imposés par l'internationalisation économique de la RPC. Cette analyse permet alors de saisir comment les changements institutionnels ont été, dans la pratique, détournés, les mesures libérales ayant conduit à un renforcement du rôle des autorités locales et provinciales sur l'ensemble de la filière.

\subsection{Les vêtements trop larges de l'industrie sucrière chinoise}

Le sucre est un produit agricole majeur, utilisé à la fois comme bien de consommation final et intermédiaire. Les accords préférentiels conservent une place prépondérante dans le commerce mondial (CNUCED), avec par exemple l'utilisation massive de subsides dans les pays développés. Ainsi, les subventions à l'exportation de l'UE (notamment les réexportations en provenance des pays ACP) ont poussé le Brésil, la Thaïlande et l'Australie à saisir l'OMC, qui leur a donné gain de cause. Cette décision ne constitue pourtant que l'une des raisons ayant poussé l'UE à réformer sa politique sucrière : la fin du «protocole sucre » entre l'UE et les pays ACP (signé en 1975 et reconduit en 2000), l'entrée de nouveaux pays dans l'Union, la mise en place d'accords du type «tout sauf les armes » en faveur des pays les plus pauvres, etc. ont conduit l'Union à adopter des réformes qui sont entrées en vigueur le 30 juin $2006^{9}$. Ces évolutions vont entraîner des changements majeurs au niveau mondial - mais dans une direction pas encore très claire ${ }^{10}$.

Dans cette situation, le rôle international de la Chine est encore mineur. Si elle est devenue le troisième producteur mondial, ses importations demeurent modestes $(0,3$ million de tonnes en 2002, et 0,1 million en 2003), tandis que ses importations se situent autour du million de tonnes. La situation devrait là aussi évoluer rapidement, mais dans un sens difficile à prévoir. D'un côté, l'augmentation de la consommation devrait pousser la Chine à importer des volumes de sucre plus importants dans les prochaines années. D'un autre côté, les dirigeants espèrent que les réformes en cours amélioreront la productivité et la rentabilité de la filière. À cet égard, ils estiment que la condamnation de l'UE à l'OMC se traduira par une diminution significative de ses exportations, conduisant à terme à une hausse des prix mondiaux de $15 \%$ à $20 \%$ (Xinhua, 2003). Ce simple effet mécanique permettrait alors au sucre chinois de devenir compétitif sur le marché mondial. C'est dans cet espoir que le Guangxi cherche à stimuler la production et que certains opérateurs étrangers ont investi dans la province ${ }^{11}$, en profitant de la mise en place des réformes. La forte hausse des prix internationaux lors de la saison de pressage 2005-2006 a permis à la filière d'inverser la tendance des années précédentes et de devenir compétitive, mais il reste à voir si ce prix (dont dépend la rentabilité de l'industrie) pourra être

\footnotetext{
${ }^{9} \mathrm{Cf}$. «Accord Européen pour baisser de $36 \%$ le soutien aux prix du sucre », Le Monde, 24 novembre 2005, Commission Européenne, juin 2005, et Commission des Communautés Européennes, 2003.

${ }^{10}$ Ibid.

${ }^{11}$ Par exemple, avec quatre sucreries, British Sugar produit 450000 tonnes de sucre par an dans la province du Guangxi (cf. British Sugar, 2005).
} 
maintenu à moyen ou long terme ${ }^{12}$, alors que les acteurs locaux cherchent à augmenter les surfaces plantées (en opposition aux politiques provinciales) ${ }^{13}$ et que la capacité productive des sucreries a largement augmenté ces dernières années (cf. infra).

Dans cette situation confuse, la Chine a commencé à engager, dès 1997, la libéralisation de son industrie sucrière, une concession dans la perspective de son admission à l'OMC. $\mathrm{Au}$ Guangxi, cette libéralisation a été perçue comme une source d'inquiétude, proportionnelle à l'importance de la filière pour l'industrie locale. De fait, la province est l'une des plus pauvres du pays et le sucre fait vivre une part importante de sa population (environ 20 millions de personnes, sur un total de 48 millions) ${ }^{14}$. Comme indiqué précédemment, les plantations de canne occupent aussi une superficie non négligeable : environ 750000 hectares, sur une surface totale de $236000 \mathrm{~km}^{2}$, dont $80 \%$ montagneuse et largement non cultivable.

Dans ce contexte, les principaux acteurs locaux ont tenté d'obtenir du gouvernement une certaine protection de leur industrie. Les réformes ont cependant pris un tour libéral, la RPC ayant continuellement augmenté ses quotas d'importation, tandis que les taxes à l'importation passaient de $30 \%$ à $15 \%$ entre 2001 et 2004. Les enjeux de la restructuration de la filière sont donc importants. Leur compréhension nécessite d'étudier les réactions des principaux acteurs, en relation avec l'organisation initiale de la filière. La prochaine section s'attache ainsi à montrer les principaux goulets d'étranglement issus de la réorganisation récente.

\subsection{Les goulets d'étranglements, conséquence d'une croissance rapide}

Afin de conserver une plus grande clarté, les analyses qui suivent, centrées sur l'organisation de la filière et son évolution, sont limitées à ses principaux acteurs : les autorités provinciales et centrales, les gouvernements locaux, les entreprises d'État, les entreprises privées et, enfin, les paysans et leurs familles. Dans cette situation, les conditions de base étaient très simples : tandis que le secteur privé était virtuellement inexistant, toutes les autres parties avaient un intérêt à développer l'industrie sucrière. Pour une province pauvre comme le Guangxi, une production de sucre accrue signifiait des revenus accrus pour les paysans, des taxes elles aussi accrues pour les autorités locales et provinciales et, enfin, un niveau de production en augmentation constante pour les entreprises d'État. La RPC connaissant une croissance économique continuelle, le potentiel semblait illimité.

\footnotetext{
${ }^{12}$ La hausse des prix internationaux est à mettre en relation avec la hausse du prix du pétrole. Il reste que le Brésil devrait augmenter de façon importante sa production de canne à sucre et de sucre de canne dans les prochaines années, impliquant la restauration des stocks mondiaux. À long terme, le prix du sucre pourrait alors poursuivre sa baisse inexorable - ce dont semble conscient le gouvernement provincial du Guangxi, mais pas les gouvernements locaux. Par ailleurs, il est à noter que la fixation du prix international du sucre est particulièrement complexe, dans la mesure où elle dépend tout autant des phénomènes de marché que d'accords internationaux et bilatéraux. L'estimation donnée par Xinhua concernant la réévaluation du prix du sucre à la suite des changements de la politique européenne doit donc être prise avec la plus grande réserve.

${ }^{13}$ Les surfaces cultivées en canne à sucre dans la province du Guangxi sont passées de 454000 hectares en 1995 à 509000 hectares en 2000, puis à 709000 hectares en 2003 et à 748000 hectares en 2005 (cf. Guangxi tongji nianjian (Statistical Yearbook of Guangxi), 2005 et 2006).

${ }^{14}$ Avec un PIB par tête de 7196 yuans (environ 900 US\$), le Guangxi est la quatrième province (sur 31) la plus pauvre de Chine (cf. China Statistical Yearbook, 2005, p. 61).
} 
Au début des années 1990 cependant, les difficultés ont commencé à poindre. Les premiers symptômes sont apparus sous la forme d'une fluctuation de la production (cf. figure 1), entraînant elle-même une fluctuation des prix. L'offre ayant augmenté plus rapidement que la demande, les fluctuations s'expliquent en partie par la situation générale de surproduction $(\mathrm{He}, 1999)$. La situation s'aggrava encore lorsque, au même moment, le gouvernement central décida de réduire ses stocks en mettant sur le marché 2 millions de tonnes de sucre, alors que les bas prix internationaux permettaient par ailleurs aux IAA de recourir aux importations (légales ou illégales) ${ }^{15}$. Cette situation conjoncturelle mit à jour les faiblesses structurelles de l'industrie, liées à des réformes de marché incomplètes et en contradiction avec l'organisation économique locale.

En effet, l'État décida, en 1991, que le prix du sucre ne serait plus fixé administrativement mais par le marché - tandis que le prix de la canne, lui, continuait (et continue toujours) a être déterminé administrativement. Cette politique limite donc la flexibilité des sucreries, notamment face à une baisse des cours. À cela, s'ajoute de graves problèmes structurels, nombre de sucreries étant au-dessous du seuil de rentabilité et employant une technologie inadéquate : à la fin des années 1990, la capacité moyenne de pressage était de 2400 tonnes de cannes par jour, contre 5200 tonnes au Mexique et 13300 tonnes en Thaillande (Ma, 2001). En 2004, la plus grande sucrerie de la province n'avait qu'une capacité de pressage de 12000 tonnes par jour - la capacité journalière moyenne ayant cependant sensiblement augmenté pour se situer aux alentours de 4000 tonnes. Enfin, les sucreries cumulaient les problèmes habituels des entreprises d'État, tels qu'un personnel pléthorique, des dettes impossibles à rembourser, etc. La plupart perdaient donc de l'argent, alors que le mot d'ordre du gouvernement central était justement de réformer les entreprises d'État inefficientes. En conséquence, les entreprises ont multiplié l'utilisation des «papiers blancs », des reconnaissances de dettes données aux paysans mais rarement honorées. D'économiques, les problèmes menaçaient de devenir sociaux - certains paysans ayant manifesté, parfois violemment. La faible superficie des champs accroissait encore les difficultés, chaque famille disposant de moins de 1 ha en moyenne pour la culture de la canne (Jiang, 2001).

Enfin, d'autres problèmes sont liés à une certaine incertitude quant à l'occupation des sols. En théorie, les paysans sont libres de cultiver ce que bon leur semble, mais sur une terre qui demeure la propriété de l'État. Des contrats leur garantissent l'utilisation des sols à des conditions spécifiées pour une certaine période, mais ces contrats ne sont pas toujours respectés par les autorités locales, la terre pouvant être tout simplement confisquée. La plupart des terrains de golf en Chine ont ainsi été construits sur des terrains confisqués illégalement aux paysans, grâce à l'aide active des cadres locaux (Tang, 2004). La terre peut aussi faire l'objet d'une redistribution périodique, afin de tenir compte des évolutions démographiques. En conséquence, les paysans du Guangxi doivent faire face à trois types de risques : ceux liés à la monoculture, de nombreux champs étant peu adaptés

\footnotetext{
${ }^{15}$ He (1999) évalue la demande de sucre pour la saison 1990/1991 à environ 7 millions de tonnes, pour une production de 8 millions, à laquelle s'ajoutent les deux millions de tonnes injectées par le gouvernement sur le marché. Le résultat a été une chute de la production entre 1992 et 1995, conduisant à une demande en excédent et une augmentation des prix. Un nouveau cycle de croissance, parfaitement visible sur le graphique 1, a finalement débuté en 1995. Il faut ajouter ici que, du fait que la canne à sucre étant une plante pérenne, l'ajustement entre l'offre et la demande nécessite un temps assez long.
} 
à d'autres cultures que celle de la canne; ceux liés à la nature contractuelle de la terre et à la non-reconnaissance de la propriété privée; et enfin, ceux liés au non-règlement de leur production par les sucreries. Ces risques limitent fortement les investissements, tandis que les faibles superficies rendent les économies d'échelle difficiles.

Dans ces conditions délicates, des réformes étaient indispensables, d'autant que la pression internationale commençait à se faire sentir. En 1996, le gouvernement central a introduit un fond de stabilisation et modifié l'usage de la réserve, afin de réduire l'instabilité des prix. Ce premier pas était cependant insuffisant pour faire face à une situation explosive : les entreprises perdaient de l'argent, et beaucoup étaient proches de la banqueroute; les finances des gouvernements locaux, qui dépendaient des taxes versées par les sucreries, étaient dans une mauvaise passe; la situation sociale s'était dégradée - les paysans étant payés en « papiers blancs » et le gouvernement ayant décidé de diminuer le prix des cannes afin d'accroître la profitabilité des sucreries. Dans ce contexte, la deuxième partie de cet article est consacrée aux réformes et à leurs effets.

\section{Les tribulations de l'industrie sucrière en Chine : plus d'économie de marché ou plus de contrôle?}

Dans cette section, il va être montré que la mise en place de réformes d'économie de marché a paradoxalement accru le contrôle effectif des autorités locales sur l'ensemble de la filière. La démonstration porte sur les conséquences organisationnelles qui découlent de l'évolution institutionnelle introduite par les réformes de marché. Comme nous l'avons indiqué précédemment, nous chercherons à savoir si l'action des acteurs principaux peut conduire à l'établissement d'un système viable, construit autour d'un compromis entre les paradigmes technologiques, organisationnels et territoriaux. L'analyse est donc empirique. Nous commencerons par une description des principales mesures, avant de montrer leur cohérence interne.

Les réformes ont visé essentiellement à rendre les sucreries plus compétitives, tout en cherchant à protéger les intérêts des paysans. L'idée de base était de réaliser à tous les niveaux de la production des économies d'échelle, dans un cadre fordiste où l'augmentation de la production allait de pair avec l'augmentation de la consommation, en relation avec la croissance générale des revenus (lois de Engel). L'introduction de capital privé et, de façon plus limitée, étranger, a ainsi permis l'augmentation de la taille des sucreries (comme cela a été indiqué précédemment). Par ailleurs, l'accroissement des surfaces des plantations de cannes a également été recherché, mais les résultats ont ici été plus mitigés ${ }^{16}$. Dans l'un et l'autre cas, les innovations techniques et technologiques locales, qui déterminent le paradigme technologique, ont été stimulées par l'action des pouvoirs publics (paradigmes technologique et organisationnel).

De fait, pour mettre en place ces réformes, le gouvernement du Guangxi bénéficiait de trois atouts majeurs : il contrôlait effectivement la centaine de sucreries d'État de la province; il était en mesure, jusqu'à un certain point, d'imposer ces réformes; enfin, malgré des hauts et des bas,

\footnotetext{
${ }^{16}$ L'un des moyens mis en œuvre a été d'aider de nouveaux cultivateurs à s'établir sur des terres nouvellement défrichées. Ces exploitations peuvent alors dépasser 35 hectares (interviews réalisées en août 2006 avec des planteurs et des cadres locaux).
} 
la production de sucre a augmenté significativement dans le Guangxi durant les années 1990 (cf. graphique 1). D'un autre côté, les réformes devaient aussi faire face aux contradictions existantes et aux intérêts divergents des principaux acteurs : contradictions entre un discours officiel qui prônait la libéralisation, et le désir des autorités locales qui souhaitaient garder l'industrie sous leur contrôle; entre la volonté de réaliser des économies d'échelles et le vital intérêt de chaque district de garder sa sucrerie (pour des raisons fiscales). La même volonté de recourir à des économies d'échelle se heurtait aussi à l'obligation technique de presser les cannes 48 heures au maximum après qu'elles aient été coupées, alors que le système routier demeure relativement arriéré. Dans ce contexte, chaque mesure a fait l'objet, ex ante et/ou ex post, d'une (re) négociation, formelle ou informelle, entre différentes catégories d'acteurs. La forme institutionnelle et/ou organisationnelle finale apparait donc fort différente de ce qu'elle aurait dû être sur le papier ${ }^{17}$ et résulte de relations de pouvoirs, venant pallier aux déficiences du marché. Les analyses qui suivent reprennent certains résultats de ce grand marchandage, tel qu'on peut le retracer à partir de rapports officiels et d'un travail sur le terrain.

Le premier maillon touché par ces réformes a été les sucreries, les deux mots clés étant alors «privatisation » et «économies d'échelles». La stratégie a reposé sur un équilibre entre la privatisation et le regroupement des actifs. Les sucreries ont été progressivement intégrées dans une quinzaine de groupes, de tailles et de statuts différents (tels que : étatiques, étatiques avec introduction en bourse, privés - avec ou sans introduction en bourse-, joint venture avec des partenaires étrangers). Dans cette partie, nous nous attacherons à présenter les réformes introduites dans deux groupes, l'un étatique et l'autre privé, qui sont particulièrement représentatifs des changements introduits dans l'industrie. La présentation de ces réformes apparaît par ailleurs compatible avec les informations que nous avons collectées et les entretiens que nous avons réalisés dans les sucreries de quatre autres groupes de différents statuts, ainsi qu'auprès de la Guangxi Sugar Association, organisme semi public dans lequel se retrouvent les groupes et les pouvoirs publics. Les deux firmes sélectionnées produisent en outre un volume de sucre conséquent, équivalent à $25 \%$ ou $30 \%$ de la production provinciale. Ces deux exemples montrent comment, sous le principe général d'une libéralisation, les autorités ont effectivement réussi à renforcer leur contrôle sur la filière, tout en y attirant une dose de capital privé. En clair, le marché du sucre est trop important pour le laisser réguler par le marché...

La seconde partie cherche donc à vérifier jusqu'à quels points la régulation du marché est compatible avec la croissance, et montrer si les mesures favorisant la concentration industrielle s'accordent avec l'organisation territoriale. La question qui demeure est donc de savoir si le compromis qui s'est dégagé de l'action des agents débouche, ou peut déboucher, sur un paradigme territorial stable et promouvant le développement.

\subsection{La reforme d'un groupe étatique : le Laixing Sugar Group}

En 1997, 23 sucreries se sont regroupées pour former le Laixing Sugar Group ${ }^{18}$. La compagnie ainsi formée devait prendre en charge la vente du sucre, tandis que chaque

\footnotetext{
${ }^{17}$ Ce point a été parfaitement illustré, dans un contexte différent, par DiMaggio : « the success of an institutionalization project and the form that the resulting institution takes depend on the relative power of the actors who support, oppose or otherwise strive to influence » (DiMaggio, p. 13).

${ }^{18}$ Le nom est fictif. Certaines descriptions de cette section sont basées sur Pan (2003).
} 
usine assurait la production. Le but était de réaliser des économies d'échelle, notamment au niveau du marketing et de la vente, pour près d'un quart des entreprises de la province $^{19}$. Le PDG devint responsable des profits et des pertes du groupe, sous l'autorité d'un conseil d'administration. Des actions furent émises, et une commission de contrôle fut créée, afin de superviser les diverses activités du groupe.

La nouvelle compagnie ressemblait fort à ses petites sœurs occidentales, avec une assemblée d'actionnaires, un conseil d'administration, un PDG et un organe de contrôle. Le but était d'introduire une certaine transparence dans la gestion - une étape décisive dans la perspective de l'adhésion de la Chine à l'OMC. Il est cependant nécessaire de ne pas s'arrêter à ces innovations, pour étudier la composition et les fonctions de ces différents organes. Il devient alors clair que les principales réformes n'ont pas rempli leurs objectifs, résultant en une augmentation des dysfonctionnements, non seulement pour le groupe, mais pour l'industrie tout entière.

Tout d'abord, l'émission d'actions, si elle fait montre d'un effort de rationalisation, n'équivaut pas à une privatisation. L'entreprise a été divisée en 24 parts, 23 allant à chaque directeur des 23 sucreries du groupe, et la dernière à l'Office du sucre du Guangxi, organe du gouvernement provincial. De la même façon, les 9 directeurs du conseil d'administration sont tous choisis parmi les 24 actionnaires, le Président étant statutairement le directeur de l'Office du sucre. Autre détail intéressant, les 23 Directeurs sont tous nommés par l'Office, de même que le PDG du nouveau groupe. Enfin, dernière touche au tableau, l'organe de contrôle est composé de 3 membres, tous actionnaires du groupe. En conséquence, l'Office du sucre est à la tête d'une organisation pyramidale et, théoriquement, il contrôle toute la structure. La transparence nouvelle, manifeste à la lecture de l'organigramme, devient beaucoup plus fictive lorsque l'on s'attache à la pratique, et la fonction du groupe, telle qu'elle émerge des réformes, est à chercher ailleurs que dans la rationalisation et la transparence. De fait, la description précédente ne constitue que la partie visible de l'iceberg. À travers certaines décisions managériales, il est possible de retracer la fonction réelle de Laixing, pour lequel les mécanismes de marché ne jouent qu'un rôle marginal.

À la fin des années 1990, la direction de Laixing décida de construire une tour de 15 étages, épuisant de la sorte la capacité financière du groupe. Laixing fut alors dans l'incapacité de payer le sucre produit par ses 23 entreprises (qui avaient conservé un système de comptabilité indépendant après la réorganisation de 1997). Le manque de disponibilités financières du groupe mit en relief son incapacité à effectuer les tâches pour lesquelles il avait été créé : assumer la vente et le marketing pour les 23 sucreries. Dans le même temps, le prix payé par Laixing aux sucreries était souvent au-dessous du cours du marché et variait en fonction des relations personnelles que les cadres du siège social à Nanning (la capitale provinciale) entretenaient avec ceux des usines. En conséquence, pour les trois années 1999-2000 le groupe fut continuellement dans le rouge ${ }^{20}$.

Les directeurs des 23 sucreries étaient donc face à un dilemme, le groupe ne versant pas (facilement) aux usines les sommes qu'il leur devait (sommes de toutes les façons inférieu-

\footnotetext{
${ }^{19}$ Le nombre de sucreries dans la province est actuellement d'environ 95, en légère diminution par rapport au milieu des années 1990, où elles étaient à peu près 110. Les 23 sucreries assurent 15 à $20 \%$ de la production totale de sucre de la province.

${ }^{20} \mathrm{Il}$ n'a pas été possible de collecter des données financières pour les années suivantes.
} 
res à ce que la vente de sucre aurait rapporté sur le marché). Deux alternatives s'offraient à eux. D'une part, ils pouvaient recourir à l'endettement, en augmentant l'émission de «tickets blancs »- une situation qui leur permettait d'attendre d'éventuels transferts financiers du groupe, mais qui pouvait aussi conduire à des problèmes sociaux graves. L'endettement pouvait aussi les acculer à la banqueroute (légale depuis 1988), ce que ni les autorités locales, ni les directeurs (en raison de leurs liens avec les autorités locales - cf. infra) ne pouvaient accepter. Cette solution paraissait donc peu praticable sur le moyen ou long terme.

D'autre part, les directeurs pouvaient choisir de conserver, ou de recréer, un département de marketing, en charge de la promotion et de la vente. Cette solution a de fait été adoptée par chacune des 23 sucreries. Le groupe devint alors une coquille vide, avec pour principales fonctions de distribuer des positions à ses clients et d'aider l'Office du sucre à renforcer sa position face à d'autres organisations concurrentes (cf. infra). Cette évolution s'accompagna aussi d'externalités négatives, créant par exemple des distorsions dans les prix du marché. En conséquence, la réforme n'aboutit qu'à créer une entité administrative supplémentaire, une sorte d' "éléphant blanc » seulement capable d'absorber des ressources. L'introduction de règles de marché et d'un système de gestion plus clair au sein d'un groupe étatique a donc échoué, (partiellement) à cause de l'impossibilité de séparer marché et bureaucratie ${ }^{21}$. Les intérêts particuliers de l'Office du sucre semblent avoir prévalu sur la réussite du programme de réformes, mais la réalité, plus nuancée, se perçoit mieux en analysant les fonctions de l'Office du sucre. Cela fait l'objet du second point de cette section.

\subsection{Jeux de pouvoir et économie de marché : \\ l'Office du Sucre et la tentative de contrôle de la filière}

La section II.1. a montré que l'Office du sucre avait, en théorie au moins, une position clé dans l'organisation de la filière. La présentation des fonctions de l'Office confirme encore cette impression : il participe à l'élaboration des politiques sucrières au niveau provincial; il est responsable pour créer ou fermer les sucreries d'État du Guangxi et pour sélectionner leurs cadres (et ce, pas seulement pour les 23 usines du groupe analysé précédemment); enfin, il a la responsabilité d'accorder les autorisations nécessaires aux sucreries non étatiques (en particulier aux entreprises privées) de commencer ou de continuer leur production. L'Office est donc juge et partie, implantant les décisions, mais étant aussi partie prenante dans la production, avec un pouvoir discrétionnaire - et même exorbitant - dans l'organisation de la filière. Cependant, ce pouvoir est contrebalancé par celui d'organisations concurrentes.

Le schéma 1 (page suivante) montre de fait une double structure pyramidale, la première étant économique et la seconde gouvernementale. En théorie, l'Office du sucre et son responsable contrôlent la centaine de sucreries d'État de la province. Cependant, ce contrôle n'est pas toujours aussi étroit qu'il y paraît, chaque sucrerie étant supervisée par la branche locale de l'Office, partie intégrante du gouvernement local. Autrement dit, ces branches locales ne sont pas de simples extensions de l'Office du sucre provincial, elles

${ }^{21}$ Une conclusion compatible avec les analyses de Jean Oi et sa notion de «local state corporatism » (Oi, 1996). Voir aussi Augustin-Jean, 2002. 
Schéma 1 : organigramme simplifié de l'appareil gouvernemental de l'organisation de l'industrie sucrière en Chine

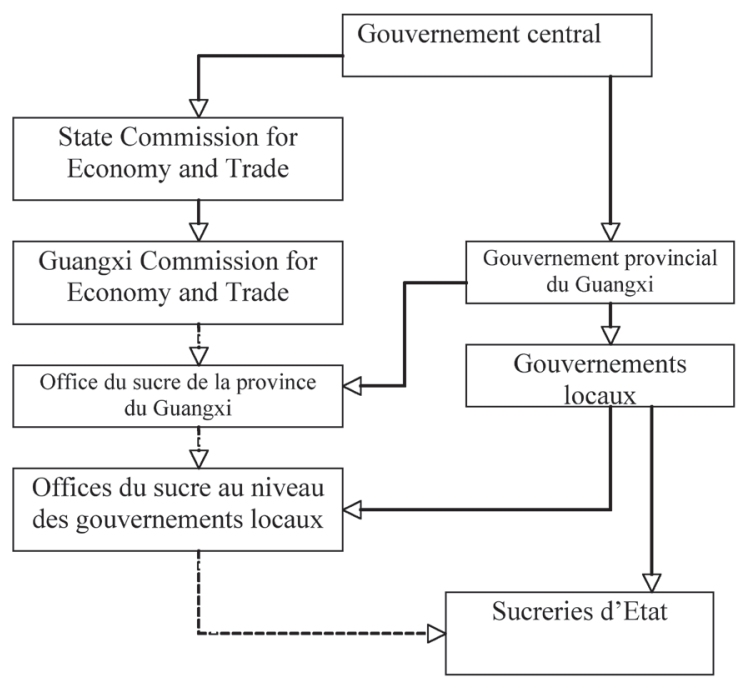

sont avant tout les organes des gouvernements locaux (les flèches en pointillé du schéma indiquant des liens de dépendance plus faibles que les flèches pleines). Il est aussi patent que les gouvernements locaux cherchent à maintenir des relations étroites avec les entreprises situées sur leur territoire (y compris les entreprises privées, comme nous le verrons dans la dernière section), car ces dernières sont les principales sources de revenus par les impôts qu'elles acquittent. Elles sont aussi la clé de la stabilité sociale, dont dépendent les cadres pour leur promotion.

Ainsi, la proximité géographique et administrative procure un instrument de contrôle puissant aux gouvernements. Car si l'Office peut nommer ou révoquer les directeurs des sucreries d'État (dans ce sens, la création de Laixing a seulement renforcé le pouvoir que l'Office a sur les 23 usines du groupe), ces responsables dépendent encore davantage des relations qu'ils ont avec les cadres locaux (en particulier pour leur carrière). Ceci explique pourquoi un département marketing a pu être recréé dans chacune des usines du groupe (au détriment de ce dernier), le sort de leur directeur étant davantage lié à celui de sa localité qu'à la pérennité de Laixing ${ }^{22}$.

Le contrôle de l'Office est également affaibli par une asymétrie d'information, qui non seulement renforce le déséquilibre en faveur des gouvernements locaux, mais a aussi des conséquences sur la gestion et l'organisation de toute la filière. En effet, les informations et les statistiques concernant les entreprises sont d'abord collectées et compilées au niveau local, avant d'être transmises aux échelons administratifs supérieurs - dont l'Office du sucre. Les statistiques, dans ce domaine comme dans d'autres, font alors l'objet de distorsions importantes, les cadres locaux manipulant souvent les données avant de les transmettre aux échelons administratifs supérieurs en fonction de leur intérêt, de celui de leur communauté, ou par rapport à des objectifs politiques prédéterminés ${ }^{23}$. En conséquence, les décisions prises par l'Office du sucre sont souvent basées sur des informations erronées ou trop générales. Par exemple, il est patent que les autorités locales cherchent systé-

\footnotetext{
${ }^{22}$ Ce principe de « double hiérarchie » est commun à toute l'administration chinoise. Pour être encore plus complet, il faudrait aussi ajouter le rôle du Parti communiste, dont les responsables locaux ont souvent un pouvoir de décision plus important que celui des technocrates et des cadres locaux. Cf. Augustin-Jean, 2000.

${ }^{23}$ Interviews, août 2005 et 2006 avec des cadres provinciaux et locaux, et avec des responsables de sucreries. Des exemples chiffrés de distorsion statistique sont fournis pour le district de Yong'an (province du Fujian) dans Augustin-Jean, 2000 .
} 
matiquement à protéger leurs entreprises ${ }^{24}$. Plus généralement encore, certaines fonctions de l'Office du sucre ont été déléguées aux branches locales, ce qui affaiblit davantage sa position au profit de ces branches.

En clair, les changements introduits dans les entreprises d'État à la fin des années 1990 ont renforcé deux caractéristiques majeures de l'économie chinoise. Premièrement, Ils ont accentué la position centrale des gouvernements locaux, principaux bénéficiaires des mesures économiques post-1978 (Augustin-Jean, 2002; Oi, 1995). Ces derniers, à partir de cette date, ont été progressivement en mesure d'implanter leur politique économique, ce qui a eu alors des conséquences positives sur le développement du pays. Aujourd'hui cependant, cette même flexibilité constitue un facteur de blocage, le gouvernement central peinant à imposer ses mesures de contrôle macroéconomiques au niveau local ${ }^{25}$. Deuxièmement, l'obligation faite aux entreprises d'État de réaliser des profits et leur autonomie accrue en matière de gestion ont conduit à un éclatement du processus décisionnel qui, échappant toujours - au moins partiellement - aux sucreries et aux groupes nouvellement créés, est devenu le produit des luttes de pouvoir et des négociations complexes entre les autorités locales, le gouvernement provincial (qui, entre autre, fixe le prix de la canne à sucre) et l'Office du sucre ${ }^{26}$. Cette organisation influe largement sur le coût de production des entreprises d'État et donc, sur leur profitabilité. Comme les sucreries d'État restent dominantes dans la province (assurant environ $40 \%$ de la production), cela affecte en retour le mécanisme de fixation du prix et donc, la compétitivité de l'industrie sucrière chinoise dans son ensemble. Malgré l'introduction de règles d'économie de marché, les jeux bureaucratiques et de pouvoir sont donc au moins aussi importants pour la fixation des prix que l'équilibre virtuel entre l'offre et la demande.

L'industrie sucrière du Guangxi reste donc vulnérable aux importations, et les réformes introduites dans les entreprises d'État à la fin des années 1990 n'ont pas réduit de façon significative les problèmes systémiques propres à la filière et à l'organisation économique chinoise. Ce commentaire est particulièrement pertinent, alors qu'un autre maillon de cette filière - les paysans - est aussi affecté par cette structure. Ce point fait l'objet de la dernière section, dans laquelle sont examinés la mise en place d'un autre type de réforme - la privatisation - ainsi que les relations entre les entreprises, les paysans et les cadres locaux.

\subsection{La privatisation, l'économie de marché et le bureaucrate}

Que la réforme des entreprises d'État ait échoué à introduire des règles transparentes peut paraître logique, et même constituer une incitation à introduire, par une politique de privatisation, davantage de marché. La privatisation a d'ailleurs été testée sur une grande échelle : certaines sucreries ont été cédées à des groupes privés ou étrangers, d'autres ont

${ }^{24}$ Dans le cadre de cette recherche, un travail de terrain a été effectué dans quatre districts ruraux, ayant au total une vingtaine de sucreries sur leur territoire. Des entretiens ont été réalisés dans une dizaine d'entre elles. Leur importance vitale pour les gouvernements locaux a été constatée dans tous les cas.

${ }^{25}$ Alors que Pékin tente actuellement de freiner la croissance afin d'éviter les phénomènes de surchauffe et les boulets d'étranglement (notamment au niveau énergétique), les districts poussent à un développement maximal. À ce sujet, la lecture de la presse locale et nationale offre un contraste particulièrement saisissant.

${ }^{26}$ L'examen de documents internes à certains groupes (différents de celui examiné dans cet article) semble par ailleurs indiquer que si l'accent est bien mis à la rentabilité et aux profits, le pouvoir de décision échappe en grande partie au conseil d'administration qui ne fait généralement qu'entériner des décisions déjà prises. 
été regroupées et cotées à la bourse de Shenzhen ou de Shanghai (impliquant l'entrée du capital privé dans des groupes qui demeurent étatiques), etc.

La privatisation est perçue par les responsables provinciaux comme un mal nécessaire : elle apporte des capitaux; elle pallie à la mauvaise santé financière des entreprises d'État ; elle offre aussi de meilleures perspectives aux autorités locales, coincées par le besoin de refinancement d'entreprises qui leur sont vitales. La recapitalisation des sucreries, à la suite des privatisations, permet ainsi d'éponger les «tickets blancs » et de desserrer la pression sociale. En contrepartie, la privatisation retire potentiellement à l'administration un moyen d'action sur un pan important de l'économie. La dernière section de cet article analyse comment les autorités locales ont pourtant réussi à conserver leur contrôle sur la filière, tout en devant composer avec le secteur privée ${ }^{27}$. Les paysans, dans le Guangxi comme ailleurs, ayant également acquis un contre-pouvoir limité, il est important d'étudier leur influence dans l'évolution du système. Leur position dans l'organisation locale ne différant pas fondamentalement lorsque les sucreries sont étatiques ou privées, les analyses qui suivent ont une portée plus générale que la simple analyse du secteur privé. Il sera donc alors possible de voir si le nouveau paradigme territorial qui s'est mis en place du fait de l'action des agents semble viable à moyen ou à long terme, ou si de nouvelles évolutions sont nécessaires à la survie de l'industrie.

La méthodologie suivie est la même que précédemment, et part d'un cas concret, celui d'un groupe privé que nous appellerons Taifeng ${ }^{28}$. Ses activités sont centrées sur la production de sucre et de produits dérivés (alcool, engrais, cartonnages, etc.), mais se sont diversifiées dans l'immobilier, l'hôtellerie et les transports. Le groupe constitue l'une des 100 plus larges entreprises privées de Chine, employant plus de 2000 personnes. Sa production de sucre pour la saison 2003/2004 se montait à 200000 tonnes, répartie dans quatre sucreries. Il n'est pas neutre de noter que le PDG de Taifeng occupe parallèlement des fonctions importantes au sein de l'appareil d'État provincial.

En 2001, Taifeng prit le contrôle d'une sucrerie d'État située dans un district rural. Cette privatisation était justifiée, aux yeux des dirigeants du district, par la mauvaise situation financière de l'entreprise qui aurait accumulé pour plus de 60 millions de yuans (soit environ 8 millions de US\$) de «papiers blancs ». La privatisation améliora la situation économique et sociale, le groupe ayant immédiatement payé les dettes et investi, la capacité de pressage passant à 10000 tonnes par jour - importante pour la province. Cette économie d'échelle s'accompagna d'un retour à la profitabilité, et l'usine fut à même de payer 36 millions de yuans de taxes au gouvernement local en 2003, soit environ $15 \%$ des ressources totales de ce dernier (interviews avec des cadres de l'entreprise et du district, juin 2004) ${ }^{29}$.

Malgré ces résultats, le groupe n'est pas libre de gérer l'entreprise comme bon lui semble, les cadres locaux conservant une emprise importante. Comme il a été déjà indiqué, il

\footnotetext{
${ }^{27}$ L'image d'une privatisation n'aboutissant qu'imparfaitement à la création d'une économie de marché vient rappeler les différences entre capitalisme et économie de marché, telles que présentées par Samir Amin (1997).

${ }^{28}$ Les données fournies dans cette section sont issues d'un travail de terrain réalisé par l'auteur en 2003 et 2004, et complétées en 2006 et 2007. L'importance de ce groupe dans le processus de privatisation de l'économie chinoise rend cet exemple particulièrement significatif, notamment en ce qui concerne la question difficile de la définition du secteur " privé » en Chine.

${ }^{29}$ Les documents que nous avons collectés dans d'autres districts montrent que les taxes payées par les sucreries peuvent représenter plus de $50 \%$ du budget local.
} 
est vital pour les gouvernements locaux que les paysans obtiennent un niveau minimum de revenus, et que, dans le même temps, les entreprises deviennent les plus rentables possible. Cette double nécessité a été reconnue par l'État, qui a mis en place deux types de régulations : d'une part, un prix plancher de la canne, afin de protéger les paysans; d'autre part, l'obligation pour les paysans de vendre leurs cannes à une sucrerie donnée (généralement située dans leur district ou a proximité).

Ce système est extrêmement lourd, pour les paysans comme pour les entreprises. Les paysans, bien qu'ils aient obtenu un prix minimum pour leurs cannes, ont perdu le droit de vendre au plus offrant. Leur marge de manœuvre est encore affaiblie par le fait que les services d'extension sont assurés par les sucreries, alors qu'ils étaient auparavant fournis par les gouvernements locaux : certains de ces services correspondant à une avance à rembourser en nature au moment des récoltes, la dépendance des paysans à « leur » entreprise en est largement accrue. D'un autre côté, les sucreries ne peuvent accéder au volume de cannes nécessaire à une expansion de grande envergure, limitant les améliorations technologiques et les économies d'échelles. Il est clair que la libre concurrence ne peut jouer ni pour les paysans, ni pour les entreprises, ce qui n'est satisfaisant pour personne. L'augmentation générale des capacités de pressage (cf. supra) se traduit donc par une compétition accrue entre sous unités territoriales (les districts ruraux), du fait que ces dernières sont souvent dans l'incapacité de produire suffisamment de cannes pour que leurs sucreries tournent à pleine capacité ${ }^{30}$. Malgré la réglementation, les sucreries cherchent donc à acheter de façon illégale les cannes produites dans les districts voisins ${ }^{31}$.

Le gouvernement hérite donc de la lourde tâche de fixer un juste prix (fair price). La pratique a montré combien il était difficile de trouver administrativement ce prix d'équilibre, tandis que nombre de chercheurs chinois ont indiqué que le marché ne pouvait à lui seul apporter de solution : une dérégulation tendrait à accroître les instabilités, au moins à court terme, ce que les autorités à tous les niveaux cherchent à empêcher à tout prix (les instabilités économiques conduisant à des instabilités sociales). Le prix artificiellement bas du sucre sur le marché mondial accentue encore davantage la pression ${ }^{32}$.

Les gouvernements locaux ne sont pas les principaux acteurs de ce jeu politique. Ils y ont cependant un intérêt vital, et peuvent manipuler les règles au niveau local, grâce à leur position d'arbitre intéressé. De façon plus directe, ils exercent aussi leur pouvoir en accordant, de façon discrétionnaire, permis et licences, et en effectuant les redistributions de terres. Leurs cadres, au premier rang desquels ceux des branches locales de l'Office du sucre, aident également les entreprises en allant « motiver» les paysans à produire un certain volume de cannes, fixé administrativement. Les carrières de ces cadres étant liées à la réalisation de ces objectifs (sans cesse revus à la hausse), ces missions de propagande se révèlent efficaces, d'autant qu'elles sont relayées sur le terrain par certains paysans qui occupent la fonction de chefs d'équipe et représentent le gouvernement local, ou de sur-

\footnotetext{
${ }^{30}$ L'augmentation des surfaces cultivées en canne ne permet donc pas de suivre la croissance de la capacité générale de pressage. La surcapacité pour l'ensemble des sucreries de la province est évaluée à $33 \%$ par les acteurs eux-mêmes.

${ }^{31}$ Malgré les interventions de la police qui patrouille aux frontières des districts. Des mesures sont actuellement testées pour tenter d'arrêter le phénomène.

${ }^{32}$ Les forts prix internationaux pour la saison 2005/2006 apportent une bouffée d'oxygène, mais pour combien de temps? Sur ce point, cf. note 12 .
} 
veillants qui organisent la production et la récolte pour les entreprises (ces derniers sont alors salariés des sucreries, selon des conditions variables aux entreprises).

Il existe donc des mécanismes qui permettent aux autorités locales et aux entreprises de stimuler la production, en intervenant directement sur les décisions des paysans. Les autorités locales sont à la fois juges et parties de ce jeu économique, mais les responsables des entreprises sont également dans une position favorable, du fait de leur proximité avec les cadres des gouvernements locaux. Les membres de ces deux groupes, en dépit de visées parfois divergentes, tendent à avancer leurs objectifs communs, en particulier face aux intérêts des districts situés à proximité, ou aux tentatives des échelons administratifs supérieurs d'extraire de leur district (auquel ils sont pieds et poings liés) une part supérieure de surplus. Leur collusion affaiblit la position des paysans, qui deviennent plus vulnérables face aux pressions exercées par les entreprises et les autorités locales. Jusqu'à un certain point néanmoins : si la partialité des cadres locaux est trop évidente, les paysans peuvent recourir à une action collective, éventuellement violente. Ces incidents se sont produits lorsque les exactions des autorités locales et/ou les « tickets blancs » deviennent trop généralisés ${ }^{33}$, ou si le prix de la canne descend au-dessous d'un certain seuil. En outre, les paysans peuvent sortir du système en se mettant à produire d'autres produits (mûriers et vers à soie par exemple).

La collusion entre les autorités locales et les entreprises peut sans doute être mieux illustrée à travers un simple exemple (qui pourrait être multiplié). Comme indiqué précédemment, les fonctions des sucreries incluent la fourniture de services d'extensions aux agriculteurs. Or malgré l'existence de leur « département agriculture », les entreprises privées sont plutôt mal équipées pour procurer des services de qualité aux paysans, du fait de leur faible niveau technologique. En revanche, les autorités locales, bien qu'elles manquent de capital, ont accès à l'information, grâce à des contacts réguliers avec les centres de recherches, les centres d'éducations techniques, etc. Pour ces entreprises, de bonnes relations avec les autorités locales (par d'ailleurs indispensables pour quiconque souhaite investir dans les campagnes chinoises) permettent de diminuer les coûts de transaction.

Le résultat de ces interactions entre le monde des sucreries et celui des cadres locaux est d'effacer en partie la limite entre la bureaucratie et les entreprises. Dans l'usine acquise en 2001 par Taifeng, le Directeur adjoint en charge de l'agriculture n'est autre que l'ancien secrétaire du Parti communiste de l'un des bourgs du district. Au sein de l'entreprise, il cumule les fonctions du développement de nouvelles espèces, de la gestion des champs (et si possible, de leur extension), de l'organisation de la récolte et de son transport (interview, avril 2004). Sa connaissance des rouages administratifs et des hommes lui donne un accès facile à l'information et, sans lui, l'usine aurait plus de difficultés à produire efficacement. De même, les fonctions exactes du responsable du bureau du sucre local sont plus complexes que ne le laissent supposer ses deux casquettes (l'une comme membre du gouvernement local, et la seconde comme dépendant de l'Office du sucre) :

«Ma tâche principale est d'assurer la coordination entre les paysans et les entreprises.

(...) [Mais] puisque l'entreprise est un important contribuable, le gouvernement est intéressé

\footnotetext{
${ }^{33}$ Cette situation est loin d'être unique : selon des données officielles, il y aurait eu, en 2004, plus de 74000 mouvements de protestations dans toute la Chine (Robertson, 2005). Une bonne analyse sur les mouvements paysans peut être trouvée chez Lucien Bianco (1994).
} 
par ses activités. Mon équipe doit travailler directement avec les paysans, puisqu'il est responsable pour fixer le prix des cannes. Un niveau équitable doit être trouvé entre les coûts de production de la sucrerie et le revenu des paysans. Le district n'a pas la décision finale sur la fixation des prix, mais il envoie des représentants à Nanning et participe aux négociations aboutissant à la fixation du prix » (interview, avril 2004).

C'est d'ailleurs durant cet entretien que j'ai appris que la privatisation de l'usine n'avait pas été complète, et que les intérêts du gouvernement local dans l'activité de l'entreprise étaient renforcés par ses $20 \%$ d'actions... Un schéma qui demeure la norme plutôt que l'exception.

Ces exemples montrent que les liens entre les entrepreneurs privés et les autorités locales sont étroits et complexes. La séparation entre les sphères gouvernementale et économique, objectif poursuivi par Pékin et qui, seule, permettrait l'avènement d'une économie de marché, est encore loin d'être une réalité ${ }^{34}$. Les réformes ont conduit à un système subtil, où les paysans, les secteurs privés et étatiques, l'Office du sucre et les gouvernements locaux et provinciaux sont étroitement imbriqués. Les réseaux qu'ils forment, mais encore plus la forme de ces réseaux reflètent les jeux de pouvoirs et de négociations et expliquent la structuration de la filière sucre du pays. Les autorités locales, qui contrôlent l'accès à l'information et pèsent sur l'organisation sociale, conservent ainsi une position centrale, même si elles doivent composer avec le secteur privé, et si les paysans peuvent toujours faire entendre leur voix à défaut de réellement pouvoir «sortir » du modèle qui leur est proposé ${ }^{35}$.

L'introduction du capital privé dans l'économie locale a ainsi permis d'apaiser certaines tensions, mais toutefois sans faire disparaître l'option «voice » que les paysans continuent à utiliser. Elle a également accentué la complexification de l'économie chinoise : la privatisation a bien permis le développement du marché, mais sans doute pas de l'économie de marché, au sens où l'entendent les économistes libéraux ou les cadres de l'OMC. Au contraire, le secteur privé s'est développé en s'insérant dans les interstices de l'économie publique, et en développant des réseaux avec les administrations. Dans la pratique, il devient de plus en plus difficile de distinguer entre les niveaux du gouvernement local, des entreprises d'État et du secteur privé, leurs limites devenant plus floues. Dans ce contexte, l'introduction du secteur privé n'a pas réglé la plupart des goulets d'étranglements et des contradictions que devront affronter les principaux agents de la filière afin d'assurer sa survie.

De façon plus fondamentale, l'existence d'une contradiction forte entre le paradigme technologique, qui pousse aux économies d'échelles et à l'augmentation de la taille des unités productives, et le paradigme territorial, qui tend au maintien du statu quo, voire à l'augmentation du nombre de sucreries, explique les blocages liés à la croissance de la filière. La conséquence est l'apparition d'une compétition entre sous unités territoriales qui débouche sur des relations conflictuelles qui ne peuvent être réglées de façon admi-

${ }^{34}$ Le gouvernement central tente actuellement, mais sans grand succès, de procéder à une certaine recentralisation (cf. Ehtisham et al., 2002).

${ }^{35} \mathrm{La}$ « sortie » n'est cependant pas impossible, certains paysans pouvant éventuellement changer de cultures. Cependant, les possibilités sont relativement limitées, et la vente de ces produits (jute, lin, patates douces, soie, etc.) n'est pas garantie. En outre, nous avons vu les pressions qui s'exercent pour pousser les paysans à produire des cannes. Les autorités locales mettent en avant les profits supérieurs et garantis de la canne à sucre par rapport à d'autres cultures. 
nistrative (par la bureaucratie de l'entité territoriale totale : la province). Le compromis ne peut non plus être trouvé dans l'établissement d'un prix d'équilibre (prix de marché de la canne à sucre), l'organisation spatiale dans laquelle se développe le marché ne permettant pas une institutionnalisation de ce dernier avec des règles précises, admises par les acteurs, et reproductibles dans le temps.

Dans ce sens, les investissements réalisés selon une logique fordiste nécessitent des quantités de canne à sucre en augmentation constante, ce qui se heurte aux capacités productives des territoires et à la rationalité des paysans. Le prix du sucre sur le marché international étant faible, la compétitivité de l'industrie chinoise ne peut que reposer sur des coûts de production faibles - c'est-à-dire, sur un prix de la canne (qui compte pour près de $60 \%$ du coût de production) relativement bas. Il a été examiné précédemment qu'il y a une impossibilité de réduire le prix des cannes, pour des raisons qui sont tout autant économiques que sociales et politiques. Une alternative serait une réforme institutionnelle générée par l'État, qui pousserait à l'apparition d'un nouveau paradigme organisationnel. Par exemple, une réforme fiscale pourrait apporter un élément de réponse à la concurrence infraterritoriale et favoriser les économies d'échelle au niveau des entreprises. S'il apparaît certain que le milieu qui s'est actuellement mis en place demeure instable et qu'un nouveau cycle de réformes devrait débuter dans les prochaines années, il ne semble pas que le gouvernement central envisage pour le moment une telle réforme fiscale qui conduirait à des risques politiques évidents au niveau de ses relations avec les autorités locales.

\section{Conclusion}

L'analyse permet de comprendre comment les agents, de par leur position au sein du système, sont parties prenantes de réseaux qu'ils mobilisent de façon stratégique pour prendre leurs décisions. Le résultat de l'action de ces agents aboutit à effacer partiellement les limites entre les entreprises et les bureaucraties. Les réseaux, beaucoup plus variés que de simples accords et contrats entre entreprises, contribuent fortement à structurer le territoire. La mobilisation de ces différents réseaux peut cependant accroître les instabilités, un résultat très différent de ce qui est généralement avancé par les théoriciens de la nouvelle économie institutionnelle (North, 1992). Dans cette situation caractérisée par de forts coûts de transaction pour tous les agents, tout se passe comme si les relations de marché étaient remplacées par des relations de pouvoir structurées autour des réseaux, et qui ne permettent pas l'apparition d'un marché stable.

Ainsi, alors que le gouvernement chinois a pris des mesures destinées à rationaliser la filière (et donc, à limiter les incertitudes), l'action des agents a fait évoluer cette dernière dans un sens qu'il était difficile à prévoir ex ante, augmentant les déséquilibres et les risques. Comme noté par Philippe Hugon dans un autre contexte, les mesures que les agents prennent pour se protéger des risques (et pour défendre leurs intérêts) conduisent paradoxalement à accroître ces mêmes risques (Hugon, 1995). En conséquence, la croissance de la filière s'est effectuée sur un fond de déséquilibres croissant en proportion de l'importance que prenait le sucre pour l'économie provinciale. Le cycle instabilité/réformes/instabilité devrait logiquement conduire à de nouvelles réformes, sur fond de pressions internationales directes (nécessité d'une ouverture plus grande des marchés) et indirectes (influence 
étrangère sur la rentabilité de la filière). Dans cette période de changements, non seulement en Chine, mais aussi sur le plan international - avec, notamment, la réforme de la politique sucrière de l'UE -, le futur semble des plus incertains, et les principaux acteurs de la filière ne cachent pas leur inquiétude. Si le cours du sucre, lors de la dernière saison de pressage, a apporté une bouffée d'optimisme, celle-ci semble se traduire par une forte volonté locale d'augmenter encore les surfaces plantées et la capacité de production des sucreries, ce qui pourrait créer des problèmes supplémentaires en cas de retournement (possible) de la conjoncture (d'autant que les réversibilités sont difficiles).

Cependant, cette réactivité de la filière aux changements internationaux est le signe le plus tangible de son intégration économique avec le reste du monde. Mais se pose alors la question, posée en introduction, de l'adéquation des règles de fonctionnement de l'économie chinoise avec celles de l'économie de marché. À partir des analyses précédentes, il semble clair qu'au-delà de la bonne volonté du gouvernement central, la transparence, la séparation entre l'économique, le politique et l'administratif (auquel il faudrait ajouter la domination, sur l'ensemble de ces domaines, du Parti communiste), sont loin d'être établies au niveau local. La question de la pérennité d'un modèle qui, certes, a produit une forte croissance, mais a aussi engendré des problèmes organisationnels importants qu'il semble difficile de régler, est donc posée. Le milieu qui s'est développé à partir des réformes et de l'interaction des agents est donc fortement instable, et sa mutation en milieu innovateur passe par un nouveau cycle de réformes qui n'est pas simple à mettre en place.

\section{Références}

2005. «Accord Européen pour baisser de $36 \%$ le soutien aux prix du sucre ». Le Monde, 24 novembre.

Amin, S. 1997. Capitalism in the Age of Globalization. London, Zed Book.

Audinet, P. 1998. L'état entrepreneur en Inde et au Brésil: économie du sucre et de l'éthanol. Paris, L'Harmattan.

Augustin-Jean, L., 2000. La dynamique entrepreneuriale en milieu rural en République populaire de Chine : les entreprises rurales non-agricoles dans le district de Zhangpu et la municipalité de Yong'an (Province du Fujian), 1978-1996. Nanterre, thèse de doctorat, Université Paris X Nanterre, 2000.

Augustin-Jean, L. 2002. «Développement des entreprises rurales et territoires en République populaire de Chine. Première partie : le contexte institutionnel et les réformes chinoises », Géographie, économie et société 4, 323-336.

Benko, G. 1990. « La dynamique spatiale de l'économie contemporaine », in Benko G., La dynamique spatiale de l'économie contemporaine. Paris, Editions de l'espace européen.

Bianco, L. 1994. « Campagnes : le retour des vendettas », Perspectives chinoises 24, 6-9.

British Sugar. 2005. Site internet du groupe. http://www.britishsugar.co.uk/RVE1a76e74838634c1b92e5386a1 89fda04,.aspx. consulté le 5 octobre 2005.

CNUCED. c.2003. «Informations sur le sucre», http://r0:unctad:org/infocomm/francais/sucre/plan.htm, consulté le 20 septembre 2005.

Commission des Communautés européennes. 2003. «Vers une réforme de la politique sucrière de l'Union européenne. Synthèse des travaux d'analyse d'impact ». Bruxelles, Document de travail des services de la commission. 
Commission Européenne. 2005. Le secteur Européen du sucre. Son importance et son avenir. Bruxelles, Commission Européenne, juin.

Crévoisier, O. 2004. «The Innovative Milieus Approach: Towards a Territorialized Understanding of the Economy? ». Economic Geography 80(4), 367-379.

Daviron, B. et Ponte, S. 2006. The Coffee Paradox. Global Markets, Commodity Trade and the Elusive Promise of Development. London, Zed Book.

DiMaggio, P. 1988. «Interest and Agency in Institutional Theory », in ZUCKER L. G., Institutional Patterns and Organizations. Cambridge, Balligner Publishing House.

Ehtisham, A. et al. 2002. « Recentralization in China?». IMF Working Paper WP/02/168.

Gereffi, G. 1999. «International Trade and Industrial Upgrading in the Apparel Commodity Chain ». Journal of International Economics 48, 37-70.

Granovetter, M. 1985. «Economic Action and Social Structure: The Problem or Embeddedness ». American Journal of Sociology 91, 481-510.

He, L. 1999. « Guangxi zhetangye fazhan de huigu he sikao (zhaiyao) » [Reflection and thinking on developments of Guangxi sugar sector (abstract)]. http://www.gxsugar.com/zjlt/zjlt13.htm, consulté le 9 novembre 2003.

Hugon, Ph. 1995. «Systèmes productifs et institutions dans un contexte de libéralisation des économies en développement ». working paper, Université Paris X Nanterre.

Jiang, T. 2001. «Guangxi tangye zhi zhanwang » [An outlook of the Guangxi sugar industry]. http://www. gxsugar.com/zjlt/zjlt05.htm, consulté le 9 octobre 2003.

Kaplinsky, R. 2000. «Spreading the Gains from Glogalization: What Can Be Learned from Value Chain Analysis ». Institute of Development Studies. Working Paper 110.

Latour, B. 2005. Reassembling the Social. An Introduction to Actor-Network-Theory, Oxford, Oxford University Press.

Ma, Z. 2001. « Cong jiejian guowai jingyan gaige he fazhan woguo tangye » [To reform and develop the sugar industry in the region with experience learnt from other countries]. http://www.gxsugar.com/zjlt/zjlt20.htm, consulté le 9 octobre 2003.

National Bureau of Statistics. 2005. China Statistical Yearbook. Beijing, China Statistics Press.

North, D. 1992. Institutions, Institutional Change and Economic Performance. Cambridge, Cambridge University Press.

Oi, J. 1995. « The Role of the Local State in China's Transitional Economy », The China Quarterly 144, 1132-1149.

Pan, T. 2003. Les entreprises d'État en Chine dans la transition économique. Mémoire de maîtrise, université de La Rochelle, La Rochelle.

Pecqueur B. 1996. Dynamiques territoriales et mutations économiques. Paris, L'Harmattan.

Rallet, A. et Torre, A. 2004. «Proximité et localisation». Papier présenté au $4^{\text {th }}$ Congress on Proximity Economics: Proximity, Networks and Co-ordination, Marseille, 17-18 juin.

Robertson, B. 2005. « Back to the roots; testing China's own btand of provincial democracy puts the Communist Party on track to resolve civil unrest, but will reforms go far enough, asks Benjamin Robertson ». Hong Kong, South China Morning Post, 15 septembre.

Saadi, H. 2005. L'économie des matières premières, Paris, L'harmattan.

Tang, M. 2004. «Government steps up effort to guarantee farmers' rights. Cultivators who lost their fields to land requisition to receive due compensation ». China Daily, 2, April 10-11.

USDA. Mai 2005. «World Production, Supply, and Distribution. Centrifugal Sugar». http://www.fas.usda. gov/htp/sugar/2005/May \% 202005\%20PSD.pdf, consulté le 15 septembre 2005.

Williamson, O. 1985. The Economic Institutions of Capitalism. New York, The Free Press, Macmillan.

Xinhua. 2003. « EU-Subsidized Sugar is Harming Chinese Farmers: Expert ». http://www.china.org.cn/English/ NBA/74901.htm (12 septembre). 
Annexe : Carte de Chine indiquant la localisation de la province du Guangxi

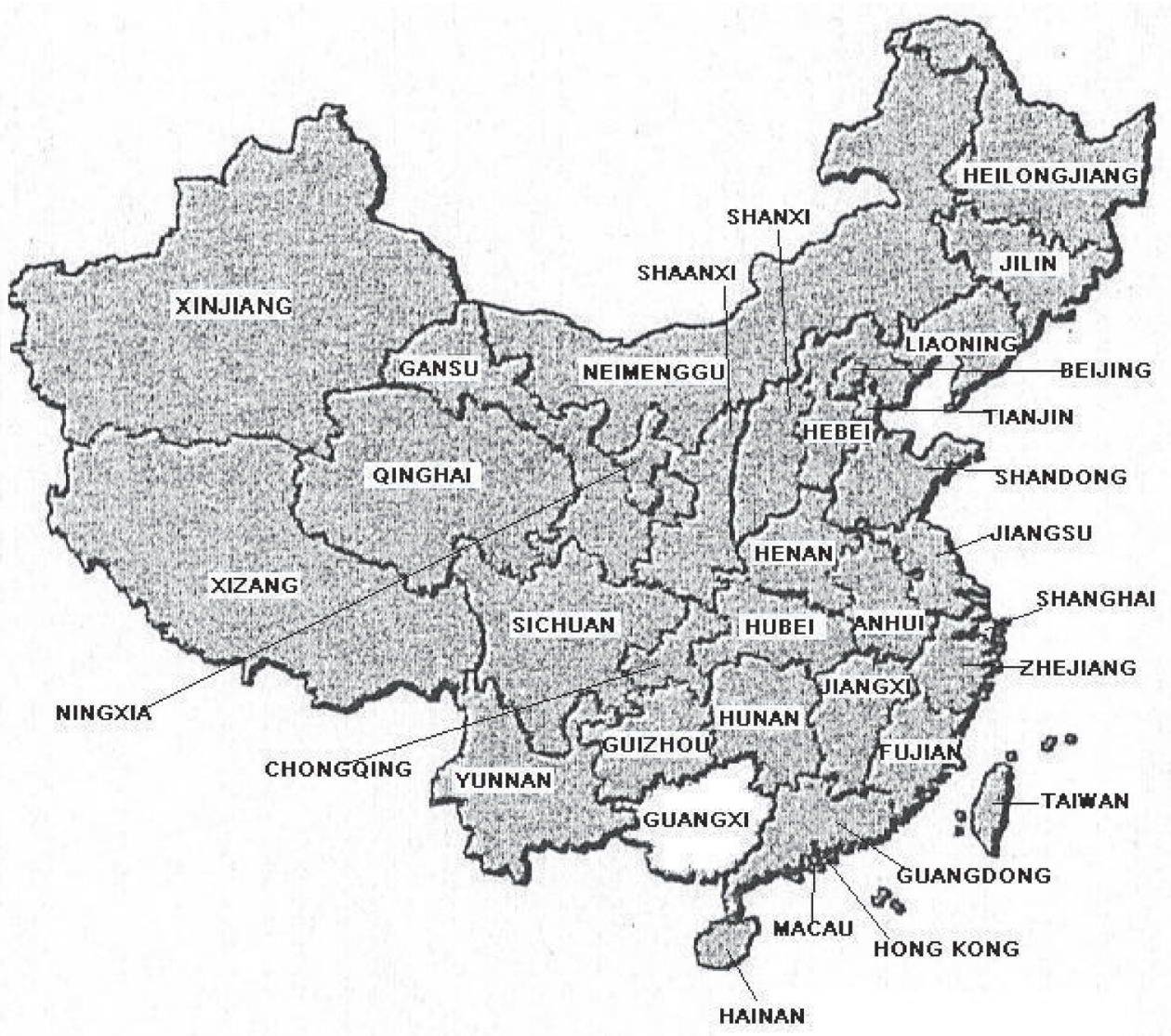


